professional perspective, (2) inform the design of future depression screening interventions or (3) inform depression screening recommendations for people with T2DM. The application of recommended qualitative evidence synthesis approaches i.e. RETREAT, GRADE-CERQual, enhances the overall robustness of the study. The protocol is available at https://hrbopenresearch.org/articles/2-26/v2.

\section{P49 A RAPID REVIEW AND PILOT STUDY OF A METHOD TO SYNTHESISE PRACTICE-BASED CASE STUDY EVIDENCE}

${ }^{1} \mathrm{~K}$ Southby* ${ }^{*}$ IJ South, ${ }^{1} \mathrm{C}$ Freeman, ${ }^{1} \mathrm{AM}$ Bagnall, ${ }^{2} \mathrm{R}$ Corcorran, ${ }^{2} \mathrm{~A}$ Pennington. ${ }^{1}$ Centre for Health Promotion Research, Leeds Beckett University, Leeds, UK; ${ }^{2}$ Department of Public Health and Policy, University of Liverpool, Liverpool, UK

\subsection{6/jech-2020-SSMabstracts. 143}

Background Case studies involve up-close, in-depth, and detailed examination of a subject (the case) and related contextual conditions. They can capture lay perspectives and provide a 'thick' description of not just public health programme outcomes but implementation processes and context. Whilst being valued by practitioners, case study evidence is often poorly regarded by policy makers as anecdotal. This is especially true for 'practice-based' case studies that involve lay people in their production. There is potential to develop robust methods to synthesise practice-based case study evidence across multiple cases. This paper reports on a project to develop and pilot an approach to synthesising practicebased case study data concerning community wellbeing.

Methods We conducted a rapid review to scope and select appropriate methods for collecting and synthesising practicebased case studies. This involved existing collections of practice-based case studies (websites and reports), backward and forward citation searching of key methodological texts, and hand searching six key academic journals. One identified method was piloted on a sample of practice-based case studies about community infrastructure for community wellbeing.

Results Eighteen publically available case study collections were reviewed and the most common information fields mapped. None of the online collections undertook further synthesis of the case studies presented. A review of forty methodological texts found that: i) practice-based case studies are commonly descriptive and often used to celebrate successes, raising questions about the depth of information that is likely to be available in a synthesis; ii) a range of methods for synthesising research-based case studies exists, with matrices/ frameworks commonly used to manage complexity, but we identified no specific methods for synthesising practice-based case study evidence; iii) traditional notions of validity and reliability are not appropriate measures of quality and alternative criteria of 'convincingness', 'authenticity', and 'plausibility' may be more useful; iv) and data collection templates can help standardise information and enhance quality of practicebased case studies and their synthesis.

Conclusion The synthesis method developed was piloted on a sample of practice-based case studies about community wellbeing. Results showed the value of synthesising practice-based case studies to produce rich data on context and processes of community projects, and information about health inequalities, but less data on outcomes. The method offers a means of supporting the production and effective utilisation of practicebased case studies in order to facilitate greater inclusion of experiential evidence from community practice into evidencebased public health.

\section{P50 DEVELOPMENT OF A NATIONAL COHORT FROM LINKED ADMINISTRATIVE DATA FOR UNDERSTANDING CHILD HEALTH AND INFORMING EARLY YEARS' POLICY}

${ }^{1} \mathrm{PM}$ Henery*, ${ }^{1} \mathrm{R}$ Dundas, ${ }^{1}$ SV Katikireddi, ${ }^{1} \mathrm{~A}$ Leyland, ${ }^{2} \mathrm{R}$ Wood, ${ }^{1} \mathrm{~A}$ Pearce. ${ }^{1} \mathrm{MRC} / \mathrm{CSO}$ Social and Public Health Sciences Unit, University of Glasgow, Glasgow, UK; ${ }^{2}$ Information Services Division, NHS National Services Scotland, Edinburgh, UK

\subsection{6/jech-2020-SSMabstracts. 144}

Background Supporting early-life health and development is one of the effective ways to improve population health and reduce inequalities. Monitoring child health and understanding its determinants is thus a policy-priority. In Scotland the community health index number (CHI), allocated to all individuals registered with a GP, presents an opportunity to link a wide range of information on mother and baby pairs from before birth and throughout the early years. We detail the development and characteristics of a national cohort from these linked data.

Methods The cohort comprised all births in Scotland, September 2009-March 2013, followed-up until March 2018. The following datasets were linked by the eData Research and Innovation Service: Scottish Morbidity (maternity) Inpatient/ Day Case Record, Scottish Birth Records, National Records of Scotland births, General/Acute Inpatient/Day Case Records, Prescribing Information System, and Child Health Systems Programme (CHSP). These contain a wide range of information on birth, demographics, socio-economic indicators, child and maternal health, and parent health-related behaviours. Socio-economic circumstances included neighbourhood deprivation and as well as individual-level measures e.g parental occupational status, relationship status of parents. Some health measures were captured continuously through registers (e.g. prescribing for attention deficit hyperactivity disorder (ADHD) in the child and depression/anxiety among the mother; childhood immunisation; hospital admissions for unintentional injury), allowing examination of health at different points of the life-course (e.g. pre and post pregnancy) Others were recorded at developmentally-appropriate ages (e.g. smoking in pregnancy, infant feeding, cognitive, physical and socio-emotional development).

Results After removing failed linkages/cleaning variable labels, the cohort consisted of 202,757 children $(97 \%$ all officially recorded births). 51.2\% were male, $3 \%$ singleton births, and $44.7 \%$ first born children. $41.8 \%$ were born to mothers $<25$ years at first live birth, $82.9 \%$ had married/cohabiting parents and $22 \%$ had a long-term unemployed mother. Among health measures (for example), 91\% had received their infant immunisations on time, $0.4 \%$ had ever been prescribed ADHD medication, and $4.9 \%$ had been admitted to hospital for an unintentional injury before school age. Most variables had no or low missing cases (e.g. mother's social class, relationship status of parents), with some exceptions (such as heights and weights).

Discussion Ability to link across administrative datasets in Scotland allows examination of child and maternal health and important determinants across the entire early years' period. We were unable to account for all emigration (as this relies on GP de-registration) and there may have been errors linking mothers to children, which may introduce bias. 